\title{
A Generalized Spectral Duality Theorem
}

\author{
Wojciech Chojnacki \\ Institute of Applied Mathematics and Mechanics, University of Warsaw, ul. Banacha 2, \\ PL-00-913 Warszawa, Poland and School of Information Science and Technology, \\ Flinders University of South Australia, GPO Box 2100, Adelaide, SA 5001, Australia
}

Dedicated to Professor Marek Burnat

\begin{abstract}
We establish a version of the spectral duality theorem relating the point spectrum of a family of *-representations of a certain covariance algebra to the continuous spectrum of an associated family of *-representations. Using that version, we prove that almost all the images of any element of a certain space of fixed points of some *-automorphism of an irrational rotation algebra via standard *-representations of the algebra in $l^{2}(\mathbb{Z})$ do not have pure point spectrum over any non-empty open subset of the common spectrum of those images. As another application of the spectral duality theorem, we prove that if almost all the Bloch operators associated with a real almost periodic function on $\mathbb{R}$ have pure point spectrum over a Borel subset of $\mathbb{R}$, then almost all the Schrödinger operators with potentials belonging to the compact hull of the translates of this function have, over the same set, purely continuous spectrum.
\end{abstract}

\section{Introduction}

Let $\Gamma=(\Omega, G, \theta, \mathbb{P})$ be a quadruple consisting of a metrizable compact space $\Omega$; a separable locally compact Abelian group $G$; a continuous $G$-action $\theta$ on $\Omega$, that is, a mapping $\theta: \Omega \times G \rightarrow \Omega$ such that $\theta(\omega, 0)=\omega$ and $\theta(\omega, g+h)=\theta(\theta(\omega, h), g)$ for $\omega \in \Omega$ and $g, h \in G$; and a Borel probability measure $\mathbb{P}$ on $\Omega$ that is $\theta_{g}$-invariant for each $g \in G$, where $\theta_{g}$ is the homeomorphism of $\Omega$ given by

$$
\theta_{g}(\omega)=\theta(\omega, g) \quad(\omega \in \Omega)
$$

Hereafter any such $\Gamma$ will be called a dynamical system. If $\Gamma=\left(\Omega, G, \theta^{(\alpha)}, m_{\Omega}\right)$ is such that $\Omega$ is a metrizable compact Abelian group, $G$ is a separable locally compact non-compact Abelian group, $\theta^{(\alpha)}$ has the form

$$
\theta^{(\alpha)}(\omega, g)=\omega+\alpha(g) \quad(\omega \in \Omega, g \in G),
$$

where $\alpha$ is a continuous one-to-one homomorphism from $G$ onto a dense subgroup of $\Omega$, and $m_{\Omega}$ is the probabilistic Haar measure on $\Omega$, then $\Gamma$ will be called a special dynamical system. 
With $\Gamma=(\Omega, G, \theta, \mathbb{P})$ a dynamical system, for $1 \leqq p<+\infty$, let $L^{p}(\Omega)$ (respectively $L^{p}(G)$ ) be the $p^{\text {th }}$ Lebesgue space based on $\mathbb{P}$ (respectively $m_{G}$ with $m_{G}$ the Haar measure on $G$ ) with norm $\|\cdot\|_{p}$.

Given a topological space $X$, let $C(X)$ be the $\mathbb{C}$-algebra of all complex continuous functions on $X$, let $C_{\mathbb{R}}(X)$ be the $\mathbb{R}$-algebra of all real functions in $C(X)$, and let $\mathscr{K}(X)$ be the $\mathbb{C}$-algebra of all complex continuous functions on $X$ with compact support.

For each $g \in G$, let $\widetilde{\theta}_{g}$ be the *-automorphism of $C(\Omega)$ given by

$$
\tilde{\theta}_{g} F=F \circ \theta_{g} \quad(F \in C(\Omega)) .
$$

We denote by $\tilde{\theta}$ the mapping $g \rightarrow \widetilde{\theta}_{g}$, which is a strongly continuous representation of $G$ into the group of *-automorphisms of $C(\Omega)$.

For each $x \in \mathscr{K}(\Omega \times G)$ and each $g \in G$, let $x_{g}$ be the element of $C(\Omega)$ given by

$$
\left(x_{g}\right)(\omega)=x(\omega, g) \quad(\omega \in \Omega) .
$$

Let $\|\cdot\|_{\infty}$ denote the supremum norm.

Equipped with a multiplication, involution, and norm defined by

$$
\begin{aligned}
(x \circ y)(\omega, g) & =\int_{G} x(\omega, h) y\left(\theta_{h}(\omega), g-h\right) d m_{G}(h), \\
x^{*}(\omega, g) & =\overline{x\left(\theta_{g}(\omega),-g\right)}, \\
\|x\|_{1} & =\int_{G}\left\|x_{g}\right\|_{\infty} d m_{G}(g) \\
(x, y & \in \mathscr{K}(\Omega \times G), \omega \in \Omega, g \in G),
\end{aligned}
$$

$\mathscr{K}(\Omega \times G)$ is a normed *-algebra. We denote by $L^{1}(\Gamma)$ the completion of $\mathscr{K}(\Omega \times G)$ in $\|\cdot\|_{1} \cdot L^{1}(\Gamma)$ is a separable Banach *-algebra, but in general is not a $C^{*}$-algebra. Setting

$$
\|x\|=\sup _{\varrho}\|\varrho(x)\| \quad\left(x \in L^{1}(\Gamma)\right),
$$

where $\varrho$ ranges over all the Hilbert space representations of $L^{1}(\Gamma)$, defines a $C^{*}$ seminorm on $L^{1}(\Gamma)$. In fact, $\|\cdot\|$ is a norm (cf. [6, Theorems 7.7.4 and 7.7.7]) and $\|x\| \leqq\|x\|_{1}$ holds for all $x \in L^{1}(\Gamma)$ (cf. [29, Theorem 25.10]). The completion of $L^{1}(\Gamma)$ in $\|\cdot\|$ is a separable $C^{*}$-algebra called the covariance algebra of $\Gamma$ or the crossed product of $C(\Omega)$ and $G$, and is denoted $C^{*}(\Gamma)$ or $C(\Omega) \times{ }_{\tilde{\theta}} G$.

Given $x \in \mathscr{K}(\Omega \times G)$ and $\omega \in \Omega$, let $\kappa_{\omega}(x)$ be the operator in $L^{2}(G)$ defined by

$$
\left(\kappa_{\omega}(x) \varphi\right)(g)=\int_{G} x\left(\theta_{g}(\omega), h\right) \varphi(g+h) d m_{G}(h) \quad\left(\varphi \in L^{2}(G), g \in G\right) .
$$

It is easily verified that for each $\omega \in \Omega$ the mapping $\kappa_{\omega}\left(x \rightarrow \kappa_{\omega}(x)\right)$ is a *-representation of $\mathscr{K}(\Omega \times G)$ in $L^{2}(G)$. The unique continuous extension of $\kappa_{\omega}$ to a *-representation of $C^{*}(\Gamma)$ in $L^{2}(G)$ will also be denoted by $\kappa_{\omega}$.

Let $\hat{G}$ be the dual group of $G$.

Given $x \in \mathscr{K}(\Omega \times G)$ and $\gamma \in \hat{G}$, let $\lambda_{\gamma}(x)$ be the operator in $L^{2}(\Omega)$ defined by

$$
\left(\lambda_{\gamma}(x) F\right)(\omega)=\int_{G} x(\omega, g)(g, \gamma) F\left(\theta_{g}(\omega)\right) d m_{G}(g) \quad\left(F \in L^{2}(\Omega), \omega \in \Omega\right) .
$$

It is easily verified that for each $\gamma \in \hat{G}$ the mapping $\lambda_{\gamma}\left(x \rightarrow \lambda_{\gamma}(x)\right)$ is a *-representation of $\mathscr{K}(\Omega \times G)$ in $L^{2}(\Omega)$. The unique continuous extension of $\lambda_{\gamma}$ to a *-representation of $C^{*}(\Gamma)$ in $L^{2}(\Omega)$ will also be denoted by $\lambda_{\gamma}$. 
Given a *-algebra $A$, let $A_{\text {sa }}$ be the self-adjoint part of $A$ and $A_{+}$be the positive part of $A$.

Given a $C^{*}$-algebra $A$, let $\mathscr{B}^{s}(A)$ be the $C^{*}$-algebra whose self-adjoint part is the strong sequential closure of $A_{\text {sa }}$ on the universal Hilbert space for $A$ (cf. [6, Subsect. 4.5.14]). As is well known, for each *-representation $\varrho$ of $A$ in a Hilbert space $H$, there is a unique sequentially normal *-representation $\varrho^{\prime \prime}$ of $\mathscr{B}(A)$ in $H$ that extends $\varrho$ (cf. [6, Theorem 3.7.7]). When $\mathscr{B}^{s}(A)$ contains a unit, which is the case, for example, when $A$ is separable, then $f(x) \in \mathscr{B}^{s}(A)$ for every $x$ in $\mathscr{B}^{s}(A)_{\text {sa }}$ and every bounded Borel function $f$ on $\mathbb{R}$ (cf. [6, Theorem 4.5.7]). Moreover, still under the assumption that $\mathscr{B}^{s}(A)$ contains a unit, $\varrho^{\prime \prime}(f(x))=f\left(\varrho^{\prime \prime}(x)\right)$ for every $x$ in $\mathscr{B}^{\mathrm{s}}(A)_{\text {sa }}$, every bounded Borel function $f$ on $\mathbb{R}$, and every *-representation $\varrho$ of $A$. In fact, given $x \in \mathscr{B}^{s}(A)_{\mathrm{sa}}$ and a ${ }^{*}$-representation $\varrho$ of $A$, the set of those bounded Borel functions $f$ on $\mathbb{R}$ for which $\varrho^{\prime \prime}(f(x))=f\left(\varrho^{\prime \prime}(x)\right)$ contains all bounded continuous functions on $\mathbb{R}$ and is strongly sequentially closed. Therefore it coincides with the set of all Borel functions on $\mathbb{R}$.

For each $x \in \mathscr{B}^{s}\left(C^{*}(\Gamma)\right)$, the function $\gamma \rightarrow\left(\lambda_{\gamma}^{\prime \prime}(x) 1,1\right)$ is Borel measurable. Indeed, the set of those $x$ in $\mathscr{B}^{s}\left(C^{*}(\Gamma)\right)$ for which the function $\gamma \rightarrow\left(\lambda_{\gamma}^{\prime \prime}(x) 1,1\right)$ is Borel measurable is weakly sequentially closed and, since $\gamma \rightarrow\left(\lambda_{\gamma}(x) 1,1\right)$ is continuous for each $x \in C^{*}(\Gamma)$, it contains $C^{*}(\Gamma)$. Thus, this set coincides with $\mathscr{B}^{s}\left(C^{*}(\Gamma)\right)$.

Given $x \in \mathscr{B}^{s}\left(C^{*}(\Gamma)\right)_{+}$, let

$$
\tau(x)=\int_{\hat{\mathbf{G}}}\left(\lambda_{\gamma}^{\prime \prime}(x) 1,1\right) d m_{\hat{\mathbf{G}}}(\gamma) .
$$

It is easily seen that $\tau$ is a $\sigma$-trace on $\mathscr{B}^{s}\left(C^{*}(\Gamma)\right)$ which in general is not faithful (see [6, Sects. 5.1.1 and 5.2.1] for relevant definitions and [19, Lemma 3.3] for the proof).

As usual, we denote by $1_{E}$ the characteristic function of the set $E$.

Let $x \in \mathscr{B}^{s}\left(C^{*}(\Gamma)\right)_{\mathrm{sa}}$ be such that $\tau\left(1_{(a, b)}(x)\right)<+\infty$ for $a, b \in \mathbb{R} \cup\{-\infty,+\infty\}$ with $a<b$. Then the spectral density function $N_{x}^{(a)}$ over $(a, b)$ is defined by

$$
N_{x}^{(a)}(\mu)=\tau\left(1_{(a, \mu]}(x)\right) \quad(\mu \in(a, b)) .
$$

$N_{x}^{(a)}$ is non-decreasing, and so the set $\mathscr{D}\left(N_{x}^{(a)}\right)$ of points of discontinuity of $N_{x}^{(a)}$ is at most countable.

Let $H$ be a Hilbert space, $T$ be a self-adjoint (bounded or unbounded) operator in $H$, and $E$ be a Borel subset of $\mathbb{R}$. We recall that $T$ is said to have pure point spectrum over $E$ if

$$
1_{E}(T)=\sum_{\mu \in E} 1_{\{\mu\}}(T)
$$

where the sum is to be interpreted in the sense of strong convergence; $T$ is said to have pure point spectrum with finite multiplicity over $E$ if the above identity is valid and, for each $\mu \in E$, the range space of the projection $1_{\{\mu\}}(T)$ is finitedimensional; and $T$ is said to have purely continuous spectrum over $E$ if $1_{\{\mu\}}(T)=0$ for each $\mu \in E$. Denote by $T_{E}$ the operator $1_{E}(T) T$. Using the identity

$$
1_{A}\left(T_{E}\right)=1_{A \cap E}(T)+\delta_{\{0\}}(A) 1_{\mathbb{R} \backslash E}(T) \quad(A \text { a Borel subset of } \mathbb{R}),
$$

where $\delta_{\{0\}}$ denotes the Dirac measure concentrated at 0 , one easily verifies that $T$ has pure point spectrum over $E$ if and only if $T_{E}$ has pure point spectrum (over $\mathbb{R}$ ).

Bellissard and Testard [5] have presented the following spectral duality theorem. 
Theorem A. Let $\Gamma=\left(\Omega, G, \theta^{(\alpha)}, m_{\Omega}\right)$ be a special dynamical system, let $a, b \in \mathbb{R}$ be such that $a<b$, and let $E$ be a Borel subset of $(a, b)$. If $x \in \mathscr{B}^{\mathrm{s}}\left(C^{*}(\Gamma)\right)_{\mathrm{sa}}$ is such that $\tau\left(1_{(a, b)}(x)\right)<+\infty$ and if, for $m_{\hat{G}^{-}}$almost all $\gamma \in \hat{G}, \lambda_{\gamma}^{\prime \prime}(x)$ has pure point spectrum with finite multiplicity over $E$, then, for $\mathbb{P}$-almost all $\omega \in \Omega, \kappa_{\omega}^{\prime \prime}(x)$ has purely continuous spectrum over E.

Kaminker and Xia [19] established another version of the spectral duality theorem, a slightly generalized variant of which, tailored to the setting of the present paper, goes as follows.

Theorem B. Let $\Gamma=(\Omega, G, \theta, \mathbb{P})$ be a dynamical system, let $a, b \in \mathbb{R}$ be such that $a<b$, and let $E$ be a Borel subset of $(a, b) \backslash \mathscr{D}\left(N_{x}^{(a)}\right)$. If $x \in \mathscr{B}^{s}\left(C^{*}(\Gamma)\right)_{\mathrm{sa}}$ is such that $\tau\left(1_{(a, b)}(x)\right)$ $<+\infty$ and if, for $m_{\hat{G}^{-}}$almost all $\gamma \in \hat{G}, \lambda_{\gamma}^{\prime \prime}(x)$ has pure point spectrum over $E$, then, for $\mathbb{P}$-almost all $\omega \in \Omega, \kappa_{\omega}^{\prime \prime}(x)$ has purely continuous spectrum over $E$.

The main purpose of the present paper is to establish a version of the spectral duality theorem that simultaneously generalizes Theorems A and B. Using that version, we prove that almost all the images of any element of a certain space of fixed points of some *automorphism of an irrational rotation algebra via standard *-representations of the algebra in $l^{2}(\mathbb{Z})$ do not have pure point spectrum over any non-empty open subset of the common spectrum of those images. As another application of the spectral duality theorem, we prove that if almost all the Bloch operators associated with a real almost periodic function on $\mathbb{R}$ have pure point spectrum over a Borel subset of $\mathbb{R}$, then almost all the Schrödinger operators with potentials belonging to the compact hull of the translates of this function have, over the same set, purely continuous spectrum.

\section{The Main Result}

We begin with a simple preliminary.

Proposition 1. Let $E$ be a Borel subset of $\mathbb{R}$, and let $H$ be a Hilbert space. If $T$ is a self-adjoint operator in $H$ such that, for each $\xi \in H$,

$$
\lim _{T \rightarrow \infty} \frac{1}{2 T} \int_{-T}^{T}\left|\left(1_{E}(T) e^{i t T} \xi, \xi\right)\right|^{2} d t=0,
$$

then $T$ has purely continuous spectrum over $E$.

Proof. The proof proceeds along the same lines as that of a well known theorem of Wiener (cf. [24, Theorem 5.6.9]).

Given $\xi \in H$, let $\eta_{\xi}$ be the spectral measure of $T$ associated with $\xi$, that is,

$$
\eta_{\xi}(A)=\left(1_{A}(T) \xi, \xi\right) \quad(A \text { a Borel subset of } \mathbb{R}) .
$$

Applying the operational calculus for normal operators (cf. [28, Theorem 11.12.3]) in conjunction with Fubini's theorem and adopting the convention that $\sin 0 / 0=1$, we find that, for each $T>0$,

$$
\begin{aligned}
\frac{1}{2 T} \int_{-T}^{T}\left|\left(1_{E}(T) e^{i t T} \xi, \xi\right)\right|^{2} d t & =\frac{1}{2 T} \int_{T}^{T}\left[\int_{E \times E} e^{i t\left(\mu-\mu^{\prime}\right)} d \eta_{\xi} \otimes \eta_{\xi}\left(\mu, \mu^{\prime}\right)\right] d t \\
& =\int_{E \times E} \frac{\sin T\left(\mu-\mu^{\prime}\right)}{T\left(\mu-\mu^{\prime}\right)} d \eta_{\xi} \otimes \eta_{\xi}\left(\mu, \mu^{\prime}\right) .
\end{aligned}
$$


If we let

$$
\mathscr{D}_{E}=\{(s, t) \in E \times E: s=t\},
$$

then, by (3) and Lebesgue's dominated convergence theorem,

$$
\lim _{T \rightarrow \infty} \frac{1}{2 T} \int_{-T}^{T}\left|\left(1_{E}(T) e^{i t T} \xi, \xi\right)\right|^{2} d t=\left(\eta_{\xi} \otimes \eta_{\xi}\right)\left(\mathscr{D}_{E}\right) .
$$

On the other hand, by Fubini's theorem,

$$
\left(\eta_{\xi} \otimes \eta_{\xi}\right)\left(\mathscr{D}_{E}\right)=\int_{E} \eta_{\xi}(\{\mu\}) d \eta_{\xi}(\mu)=\sum_{\mu \in E}\left|\eta_{\xi}(\{\mu\})\right|^{2} .
$$

Hence, in view of (2) and (4),

$$
\sum_{\mu \in E}\left|\eta_{\xi}(\{\mu\})\right|^{2}=0
$$

implying that $\eta_{\xi}(\{\mu\})=0$ for each $\mu \in E$. By the arbitrariness of $\xi, 1_{\{\mu\}}(T)=0$ for each $\mu \in E$.

The proof is complete.

The main result of this section is the following.

Theorem 2. Let $\Gamma=(\Omega, G, \theta, \mathbb{P})$ be a dynamical system, and let $E$ be a Borel subset of $\mathbb{R}$. If $x$ is an element of $\mathscr{B}^{s}\left(C^{*}(\Gamma)\right)_{\mathrm{sa}}$ such that $\tau\left(1_{\{\mu\}}(x)\right)=0$ for each $\mu \in E$ and if, for $m_{\hat{G}^{-}}$almost all $\gamma \in \hat{G}, \lambda_{\gamma}^{\prime \prime}(x)$ has pure point spectrum over $E$, then, for $\mathbb{P}$-almost all $\omega \in \Omega$, $\kappa_{\omega}^{\prime \prime}(x)$ has purely continuous spectrum over $E$.

Proof. Given $\varphi \in L^{1}(G)$, let $\hat{\varphi}$ be the Fourier transform of $\varphi$, that is,

$$
\hat{\varphi}(\gamma)=\int_{G} \varphi(g)(g,-\gamma) d m_{G}(g) \quad(\gamma \in \hat{G}) .
$$

Adopting a standard convention, we assume that the Haar measure on $\hat{G}$ is normalized so that

$$
\varphi(x)=\int_{\hat{G}} \hat{\varphi}(\gamma)(x, \gamma) d m_{\hat{G}}(\gamma) \quad(x \in G)
$$

whenever $\varphi \in L^{1}(G) \cap C(G)$ and $\hat{\varphi} \in L^{1}(\hat{G})$.

Let $A(G)$ be the space of the Fourier transforms of functions in $L^{1}(\hat{G})$.

For each $x \in \mathscr{K}(\Omega \times G)$, each $\varphi \in A(G) \cap \mathscr{K}(G)$, and each $g \in G$, we have

$$
\begin{aligned}
\int_{\Omega} \mid & \left.\left(\kappa_{\omega}(x) \varphi\right)(g)\right|^{2} d \mathbb{P}(\omega) \\
= & \int_{\Omega \times G \times G} x\left(\theta_{g}(\omega), h\right) \overline{x\left(\theta_{g}(\omega), h^{\prime}\right)} \varphi(g+h) \overline{\varphi\left(g+h^{\prime}\right)} d \mathbb{P} \otimes m_{G \times G}\left(\omega, h, h^{\prime}\right) \\
= & \int_{G \times G}\left[\int_{\Omega} x(\omega, h) \overline{x\left(\omega, h^{\prime}\right)} d \mathbb{P}(\omega)\right] \varphi(g+h) \varphi \overline{\left(g+h^{\prime}\right)} d m_{G \times G}\left(h, h^{\prime}\right) \\
= & \int_{G \times G \times \hat{G} \times \hat{G}}\left[\int_{\Omega} x(\omega, h) \overline{x\left(\omega, h^{\prime}\right)} d \mathbb{P}(\omega)\right](g+h, \gamma)\left(g+h^{\prime},-\gamma^{\prime}\right) \\
& \quad \times \hat{\varphi}(\gamma) \hat{\hat{\varphi}\left(\gamma^{\prime}\right)} d m_{G \times G \times \hat{G} \times \hat{G}}\left(h, h^{\prime}, \gamma, \gamma^{\prime}\right) \\
= & \int_{\hat{G} \times \hat{G}}\left(\lambda_{\gamma}(x) 1, \lambda_{\gamma^{\prime}}(x) 1\right)\left(g, \gamma-\gamma^{\prime}\right) \hat{\varphi}(\gamma) \overline{\hat{\varphi}\left(\gamma^{\prime}\right)} d m_{\hat{G} \times \hat{G}}\left(\gamma, \gamma^{\prime}\right) .
\end{aligned}
$$

Note that the use of Fubini's theorem is legitimate since $\hat{\varphi}$ is in $L^{1}(\hat{G}) \cap C(\hat{G})$ and hence the function $\left(\gamma, \gamma^{\prime}\right) \rightarrow \hat{\varphi}(\gamma) \overline{\hat{\varphi}\left(\gamma^{\prime}\right)}$ is in $L^{1}(\hat{G} \times \hat{G}) \cap C(\hat{G} \times \hat{G})$. Let $K$ be a compact 
subset of $G$ containing the support of $\varphi$. Then, for each $\omega \in \Omega$,

$$
\left|\left(\kappa_{\omega}(x) \varphi, \varphi\right)\right|^{2} \leqq m_{G}(K)\|\varphi\|_{\infty}^{2} \int_{K}\left|\left(\kappa_{\omega}(x) \varphi\right)(g)\right|^{2} d m_{G}(g) .
$$

This together with (5) yields

$$
\begin{aligned}
\int_{\Omega}\left|\left(\kappa_{\omega}(x) \varphi, \varphi\right)\right|^{2} d \mathbb{P}(\omega) \leqq & m_{G}(K)\|\varphi\|_{\infty}^{2} \int_{K \times \hat{G} \times \hat{G}}\left(\lambda_{\gamma}(x) 1, \lambda_{\gamma^{\prime}}(x) 1\right)\left(g, \gamma-\gamma^{\prime}\right) \\
& \times \hat{\varphi}(\gamma) \overline{\hat{\varphi}\left(\gamma^{\prime}\right)} d m_{G \times \hat{G} \times \hat{G}}\left(g, \gamma, \gamma^{\prime}\right) .
\end{aligned}
$$

The latter inequality implies in turn that, for each $x \in \mathscr{B}^{s}\left(C^{*}(\Gamma)\right)$,

$$
\begin{aligned}
\int_{\Omega}\left|\left(\kappa_{\omega}^{\prime \prime}(x) \varphi, \varphi\right)\right|^{2} d \mathbb{P}(\omega) \leqq & m_{G}(K)\|\varphi\|_{\infty}^{2} \int_{K \times \hat{G} \times \hat{G}}\left(\lambda_{\gamma}^{\prime \prime}(x) 1, \lambda_{\gamma^{\prime}}^{\prime \prime}(x) 1\right)\left(g, \gamma-\gamma^{\prime}\right) \\
& \times \hat{\varphi}(\gamma) \overline{\hat{\varphi}\left(\gamma^{\prime}\right)} d m_{G \times \hat{G} \times \hat{G}}\left(g, \gamma, \gamma^{\prime}\right) .
\end{aligned}
$$

In fact, by the previous argument, for each $x \in \mathscr{B}^{s}\left(C^{*}(\Gamma)\right)$ the functions $\omega \rightarrow\left(\kappa_{\omega}^{\prime \prime}(x) \varphi, \varphi\right)$ and $\left(\gamma, \gamma^{\prime}\right) \rightarrow\left(\lambda_{\gamma}^{\prime \prime}(x) 1, \lambda_{\gamma^{\prime}}^{\prime \prime}(x) 1\right)$ are Borel measurable. Moreover, by Lebesgue's dominated convergence theorem, the set of those $x$ in $\mathscr{B}^{s}\left(C^{*}(\Gamma)\right)$ for which (7) holds is strongly sequentially closed, and, by $(6)$, contains $C^{*}(\Gamma)$. It therefore coincides with $\mathscr{B}^{s}\left(C^{*}(\Gamma)\right)$.

Let $E$ be Borel subset of $\mathbb{R}$ and $x \in \mathscr{B}^{s}\left(C^{*}(\Gamma)\right)_{\mathrm{sa}}$ be such that $\tau\left(1_{\{\mu\}}(x)\right)=0$ for each $\mu \in E$ and, for $m_{\hat{G}^{-}}$-almost all $\gamma \in \hat{G}, \lambda_{\gamma}^{\prime \prime}(x)$ has pure point spectrum over $E$. We claim

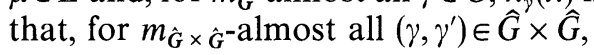

$$
\lim _{T \rightarrow \infty} \frac{1}{2 T} \int_{-T}^{T}\left(\lambda_{\gamma}^{\prime \prime}\left(1_{E}(x) e^{i t x}\right) 1, \lambda_{\gamma^{\prime}}^{\prime \prime}\left(1_{E}(x) e^{i t x}\right) 1\right) d t=0 .
$$

Let $\Delta$ be the set of those $\gamma \in \hat{G}$ for which $\lambda_{y}^{\prime \prime}(x)$ has pure point spectrum over $E$. For each $\gamma \in \Delta$, let $\left(X_{\gamma, i}\right)_{i \in \mathscr{J}_{\nu}}$ be a complete system of eigenvectors of the restriction of $\lambda_{\gamma}^{\prime \prime}(x)$ to the range space $\mathscr{R}\left(1_{E}\left(\lambda_{\gamma}^{\prime \prime}(x)\right)\right)$ of the projection $1_{E}\left(\lambda_{\gamma}^{\prime \prime}(x)\right)$ with a corresponding system $\left(\mu_{\gamma, i}\right)_{i \in \mathscr{F}_{\gamma}}$ of eigenvalues, where the index set $\mathscr{J}_{\gamma}$ has the cardinality equal to the orthogonal dimension of $\mathscr{R}\left(1_{E}\left(\lambda_{\gamma}^{\prime \prime}(x)\right)\right)$. Given $\gamma \in \Delta$ and $\varepsilon>0$, let $I_{\gamma, \varepsilon}$ be a finite subset of $\mathscr{J}_{\gamma}$ such that

$$
\left\|1_{E}\left(\lambda_{\gamma}^{\prime \prime}(x)\right) 1-\sum_{i \in I_{\gamma, \varepsilon}}\left(1_{E}\left(\lambda_{\gamma}^{\prime \prime}(x)\right) 1, X_{\gamma, i}\right) X_{\gamma, i}\right\|_{2}<\varepsilon .
$$

Notice that $\left(1_{E}\left(\lambda_{\gamma}^{\prime \prime}(x)\right) 1, X_{\gamma, i}\right)=\left(1, X_{\gamma, i}\right)$ whatever $\gamma \in \Delta$ and $i \in \mathscr{J}_{\gamma}$. Thus, for any $\gamma, \gamma^{\prime} \in \Delta$, any $\varepsilon>0$, and any $t \in \mathbb{R}$, we have

$$
\begin{aligned}
& \mid\left(\lambda_{\gamma}^{\prime \prime}\left(1_{E}(x) e^{i t x}\right) 1, \lambda_{\gamma^{\prime}}^{\prime \prime}\left(1_{E}(x) e^{i t x}\right) 1\right) \\
& -\sum_{(i, j) \in I_{\gamma, \varepsilon} \times I_{\gamma^{\prime}, \varepsilon}} e^{i t\left(\mu_{\gamma, i}-\mu_{\gamma^{\prime}, j}\right)}\left(1, X_{\gamma, i}\right) \overline{\left(1, X_{\gamma^{\prime}, j}\right)}\left(X_{\gamma, i}, X_{\gamma^{\prime}, j}\right) \\
& \leqq\left|\left(\lambda_{\gamma}^{\prime \prime}\left(1_{E}(x) e^{i t x}\right)\left(1_{E}\left(\lambda_{\gamma}^{\prime \prime}(x)\right) 1-\sum_{i \in I_{\gamma, \varepsilon}}\left(1, X_{\gamma, i}\right) X_{\gamma, i}\right), \lambda_{\gamma}^{\prime \prime}\left(1_{E}(x) e^{i t x}\right) 1\right)\right| \\
& +\mid\left(\sum_{i \in I_{\gamma, \varepsilon}}\left(1, X_{\gamma, i}\right) \lambda_{\gamma}^{\prime \prime}\left(1_{E}(x) e^{i t x}\right) X_{\gamma, i},\right. \\
& \left.\lambda_{\gamma^{\prime}}^{\prime \prime}\left(1_{E}(x) e^{i t x}\right)\left(1_{E}\left(\lambda_{\gamma^{\prime}}^{\prime \prime}(x)\right) 1-\sum_{j \in I_{\gamma^{\prime}, \varepsilon}}\left(1, X_{\gamma^{\prime}, j}\right) X_{\gamma^{\prime}, j}\right)\right) \mid \\
& \leqq \varepsilon+\varepsilon\left\|_{i \in I_{\nu, \varepsilon}}\left(1, X_{\gamma, i}\right) X_{\gamma, i}\right\|_{2} \leqq \varepsilon(2+\varepsilon) .
\end{aligned}
$$


Given $\mu \in E$ and $\gamma \in \Delta$, let

$$
J_{\mu, \gamma}=\left\{i \in \mathscr{J}_{\gamma}: \mu=\mu_{\gamma, i}\right\} .
$$

Plainly, for each $\mu \in E$ and each $\gamma \in \Delta$,

$$
\left(\lambda_{\gamma}^{\prime \prime}\left(1_{\{\mu\}}(x)\right) 1,1\right)=\left\|\lambda_{\gamma}^{\prime \prime}\left(1_{\{\mu\}}(x)\right) 1\right\|_{2}^{2}=\left\|1_{\{\mu\}}\left(\lambda_{\gamma}^{\prime \prime}(x)\right) 1\right\|_{2}^{2}=\sum_{i \in J_{\mu, \gamma}}\left|\left(1, X_{\gamma, i}\right)\right|^{2} .
$$

Hence, by (1) and the assumption, for each $\mu \in E$ the set

$$
\Gamma_{\mu}=\left\{\gamma \in \Delta:\left(1, X_{\gamma, i}\right)=0 \text { for } i \in J_{\mu, \gamma}\right\}
$$

is of full measure in $\hat{G}$. Given $\gamma \in \Delta$ and $\varepsilon>0$, let

$$
\Delta_{\gamma, \varepsilon}=\bigcap_{i \in I_{\gamma, \varepsilon}} \Gamma_{\mu_{\gamma, i}} .
$$

Clearly, $\Delta_{\gamma, \varepsilon}$ is also of full measure in $\hat{G}$.

Fix $\gamma \in \Delta$ and $\gamma^{\prime} \in \Delta_{\gamma, \varepsilon}$ arbitrarily. Note that, if $\mu_{\gamma, i}=\mu_{\gamma^{\prime}, j}$ for some $(i, j) \in I_{\gamma, \varepsilon}$ $\times I_{\gamma^{\prime}, \varepsilon}$, then, since $\gamma^{\prime} \in \Gamma_{\mu_{\gamma, i}}$, we have that $\left(1, X_{\gamma^{\prime}, j}\right)=0$. Therefore, if we let

$$
\mathscr{A}_{\gamma, \gamma^{\prime}, \varepsilon}=\left\{(i, j) \in I_{\gamma, \varepsilon} \times I_{\gamma^{\prime}, \varepsilon}: \mu_{\gamma, i} \neq \mu_{\gamma^{\prime}, j}\right\},
$$

then, for each $t \in \mathbb{R}$,

$$
\begin{aligned}
& \sum_{(i, j) \in I_{\gamma, \varepsilon} \times I_{\gamma^{\prime}, \varepsilon}} e^{i t\left(\mu_{\gamma, i}-\mu_{\gamma^{\prime}, j}\right)}\left(1, X_{\gamma, i}\right) \overline{\left(1, X_{\gamma^{\prime}, j}\right)}\left(X_{\gamma, i}, X_{\gamma^{\prime}, j}\right) \\
= & \sum_{(i, j) \in \mathscr{A}_{\gamma, \gamma^{\prime}, \varepsilon}} e^{i t\left(\mu_{\gamma, i}-\mu_{\gamma^{\prime}, j}\right)}\left(1, X_{\gamma, i}\right) \overline{\left(1, X_{\gamma^{\prime}, j}\right)}\left(X_{\gamma, i}, X_{\gamma^{\prime}, j}\right) .
\end{aligned}
$$

This together with (6) implies that, for each $\gamma \in \Delta$ and each $\gamma^{\prime} \in \Delta_{\gamma, \varepsilon}$,

$$
\limsup _{T \rightarrow \infty}\left|\frac{1}{2 T} \int_{-T}^{T}\left(\lambda_{\gamma}^{\prime \prime}\left(1_{E}(x) e^{i t x}\right) 1, \lambda_{\gamma^{\prime}}^{\prime \prime}\left(1_{E}(x) e^{i t x}\right) 1\right) d t\right| \leqq \varepsilon(2+\varepsilon) .
$$

Let

$$
\tilde{\Delta}_{\gamma}=\bigcap_{n \in \mathbb{N}} \Delta_{\gamma, 1 / n} .
$$

Clearly, $\tilde{\Delta}_{\gamma}$ is of full measure in $\hat{G}$. Moreover, (8) holds for all $\gamma \in \Delta$ and all $\gamma^{\prime} \in \tilde{\Delta}_{\gamma^{\prime}}$. Let $N$ be the Borel set of those $\left(\gamma, \gamma^{\prime}\right) \in \hat{G} \times \hat{G}$ for which (8) holds. For each $\gamma \in \hat{G}$, let

$$
N_{\gamma}=\left\{\gamma^{\prime} \in \hat{G}:\left(\gamma, \gamma^{\prime}\right) \in N\right\} .
$$

Since $\tilde{\Delta}_{\gamma} \subset N_{\gamma}$ for every $\gamma \in \Delta$, it follows from Fubini's theorem that $N$ has full measure in $\hat{G} \times \hat{G}$. The claim is thus established.

The function $\left(\gamma, \gamma^{\prime}\right) \rightarrow \hat{\varphi}(\gamma) \hat{\varphi}\left(\gamma^{\prime}\right)$ is in $L^{1}(\hat{G} \times \hat{G}) \cap C(\hat{G} \times \hat{G})$ and $K$ is compact, so, by (8) and Lebesgue's dominated convergence theorem,

$$
\begin{aligned}
& \lim _{T \rightarrow \infty} \frac{1}{2 T} \int_{-T}^{T}\left[\int_{K \times \hat{G} \times \hat{\boldsymbol{G}}}\left(\lambda_{\gamma}^{\prime \prime}\left(1_{E}(x) e^{i t x}\right) 1, \lambda_{\gamma^{\prime}}^{\prime \prime}\left(1_{E}(x) e^{i t x}\right) 1\right)\left(g, \gamma-\gamma^{\prime}\right)\right. \\
& \left.\quad \times \hat{\varphi}(\gamma) \overline{\hat{\varphi}\left(\gamma^{\prime}\right)} d m_{G \times \hat{G} \times \hat{G}}\left(g, \gamma, \gamma^{\prime}\right)\right] d t=0 .
\end{aligned}
$$

This jointly with (7) implies that

$$
\lim _{T \rightarrow \infty} \frac{1}{2 T} \int_{-T}^{T}\left[\int_{\Omega}\left|\left(\kappa_{\omega}^{\prime \prime}\left(1_{E}(x) e^{i t x}\right) \varphi, \varphi\right)\right|^{2} d \mathbb{P}(\omega)\right] d t=0 .
$$


Since, for each $T>0$,

$$
\frac{1}{2 T} \int_{-T}^{T}\left|\left(\kappa_{\omega}^{\prime \prime}\left(1_{E}(x) e^{i t x}\right) \varphi, \varphi\right)\right|^{2} d t \leqq\|\varphi\|_{2}^{2}
$$

it follows from Lebesgue's dominated convergence theorem that

$$
\int_{\Omega}\left[\lim _{T \rightarrow \infty} \frac{1}{2 T} \int_{-T}^{T}\left|\left(\kappa_{\omega}^{\prime \prime}\left(1_{E}(x) e^{i t x}\right) \varphi, \varphi\right)\right|^{2} d t\right] d \mathbb{P}(\omega)=0 .
$$

Thus, for $\mathbb{P}$-almost all $\omega \in \Omega$,

$$
\lim _{T \rightarrow \infty} \frac{1}{2 T} \int_{-T}^{T}\left|\left(\kappa_{\omega}^{\prime \prime}\left(1_{E}(x) e^{i t x}\right) \varphi, \varphi\right)\right|^{2} d t=0
$$

The space $A(G) \cap \mathscr{K}(G)$ is dense in $L^{2}(G)$ and the latter space is separable. Therefore there exists a $\mathbb{P}$-null subset $N$ of $\Omega$ such that, for all $\varphi \in L^{2}(\Omega)$ and all $\omega \in \Omega \backslash N$,

$$
\lim _{T \rightarrow \infty} \frac{1}{2 T} \int_{-T}^{T}\left|\left(1_{E}\left(\kappa_{\omega}^{\prime \prime}(x)\right) e^{i t \kappa^{\prime \prime}(x)} \varphi, \varphi\right)\right|^{2} d t=0 .
$$

In view of Proposition 1, for each $\omega \in \Omega \backslash N, \kappa_{\omega}^{\prime \prime}(x)$ has purely continuous spectrum over $E$.

The proof is complete.

\section{Some Consequences}

It is clear that Theorem 2 implies Theorem B. The proof of the fact that Theorem 2 implies Theorem A is based on the following.

Proposition 3. Let $\Gamma=\left(\Omega, G, \theta^{(\alpha)}, m_{\Omega}\right)$ be a special dynamical system, and let $E$ be a Borel subset of $\mathbb{R}$. If $x \in \mathscr{B}^{\mathrm{s}}\left(C^{*}(\Gamma)\right)_{\mathrm{sa}}$ is such that, for $m_{\hat{G}^{-}}$almost all $\gamma \in \hat{G}, \lambda_{\gamma}^{\prime \prime}(x)$ has pure point spectrum with finite multiplicity over $E$, then $\tau\left(1_{\{\mu\}}(x)\right)=0$ for each $\mu \in E$.

Proof. Let $\hat{\alpha}$ be the homomorphism from $\hat{\Omega}$ to $\hat{G}$ given by

$$
(g, \hat{\alpha}(\zeta))=(\alpha(g), \zeta) \quad(\zeta \in \hat{\Omega}, g \in G) .
$$

Since $\alpha$ is one-to-one, $\hat{\alpha}(\widehat{\Omega})$ is dense in $\hat{G}$. Since $G$ is non-compact and $\sigma$-compact, $\hat{G}$ is non-discrete and metrizable (cf. [23, Theorems 12 and 29]). Thus there exists a sequence $\left(\zeta_{k}\right)_{k \in \mathbb{N}}$ of pairwise different elements of $\hat{\Omega}$ such that $\lim _{k \rightarrow \infty} \hat{\alpha}\left(\zeta_{k}\right)=0$.

For a measure space $(X, \mathfrak{M}, \mu)$ and $f \in L^{\infty}(X, \mu)$, we denote by $M_{f}$ the operator in $L^{2}(X, \mu)$ given by

$$
M_{f} \varphi=f \varphi \quad\left(\varphi \in L^{2}(X, \mu)\right) .
$$

For a non-negative operator $S$ in a Hilbert space, we denote by $\operatorname{Tr}(S)$ the trace of $S$.

For a set $E, \# E$ denotes the cardinality of $E$.

Given a subset $E$ of an Abelian group $A$ and an element $a$ of $A$, we let

$$
E+a=\{b \in A: b=e+a, e \in E\} .
$$

A direct computation shows that, for each $\zeta \in \hat{\Omega}$, each $\gamma \in \hat{G}$, and each $y \in \mathscr{B}^{s}\left(C^{*}(\Gamma)\right)$,

$$
M_{-\zeta} \lambda_{\gamma}^{\prime \prime}(y) M_{\zeta}=\lambda_{\gamma+\hat{\alpha}(\zeta)}^{\prime \prime}(y)
$$


Hence, if $x \in \mathscr{B}^{s}\left(C^{*}(\Gamma)\right)_{\mathrm{sa}}$ is such that $\lambda_{\gamma}^{\prime \prime}(x)$ has pure point spectrum with finite multiplicity over $E$ for every $\gamma$ in a set $\Delta$ of full measure in $\hat{G}$, then, for each $\mu \in E$, each $\gamma \in \Delta$, and each $n \in \mathbb{N}$,

$$
\sum_{k=1}^{n}\left(\lambda_{\gamma+\hat{\alpha}\left(\zeta_{k}\right)}^{\prime \prime}\left(1_{\{\mu\}}(x)\right) 1,1\right)=\sum_{k=1}^{n}\left(\lambda_{\gamma}^{\prime \prime}\left(1_{\{\mu\}}(x)\right) \zeta_{k}, \zeta_{k}\right) \leqq \operatorname{Tr}\left(\lambda_{\gamma}^{\prime \prime}\left(1_{\{\mu\}}(x)\right)\right)=\# J_{\mu, \gamma} .
$$

In particular, for each $\mu \in E$ and each $\gamma \in \Delta$,

$$
\lim _{n \rightarrow \infty} \frac{1}{n} \sum_{k=1}^{n}\left(\lambda_{\gamma+\hat{\alpha}\left(\zeta_{k}\right)}^{\prime \prime}\left(1_{\{\mu\}}(x)\right) 1,1\right)=0 .
$$

Let $K$ be a compact subset of $\hat{G}$. Since $\lim _{k \rightarrow \infty} \hat{\alpha}\left(\zeta_{k}\right)=0$, it follows that, for each $\mu \in E$,

$$
\begin{aligned}
\int_{K}\left(\lambda_{\gamma}^{\prime \prime}\left(1_{\mu}(x)\right) 1,1\right) d m_{\hat{G}}(\gamma) & =\lim _{n \rightarrow \infty} \frac{1}{n} \sum_{k=1}^{n} \int_{K-\hat{\alpha}\left(\zeta_{k}\right)}\left(\lambda_{\gamma}^{\prime \prime}\left(1_{\mu}(x)\right) 1,1\right) d m_{\hat{G}}(\gamma) \\
& =\lim _{n \rightarrow \infty} \int_{K} \frac{1}{n} \sum_{k=1}^{n}\left(\lambda_{\gamma+\hat{\alpha}\left(\zeta_{k}\right)}^{\prime \prime}\left(1_{\mu}(x)\right) 1,1\right) d m_{\hat{\mathbf{G}}}(\gamma) .
\end{aligned}
$$

On the other hand, by (11) and Lebesgue's dominated convergence theorem, for each $\mu \in E$, we have

$$
\lim _{n \rightarrow \infty} \int_{K} \frac{1}{n} \sum_{k=1}^{n}\left(\lambda_{\gamma+\hat{\alpha}\left(\zeta_{k}\right)}^{\prime \prime}\left(1_{\mu}(x)\right) 1,1\right) d m_{\hat{G}}(\gamma)=0 .
$$

Hence, for each $\mu \in E,\left(\lambda_{\gamma}^{\prime \prime}\left(1_{\mu}(x)\right) 1,1\right)=0$ for $m_{\hat{G}^{-}}$-almost all $\gamma \in K$ and, in view of the arbitrariness of $K,\left(\lambda_{\nu}^{\prime \prime}\left(1_{\mu}(x)\right) 1,1\right)=0$ for $m_{\hat{G}^{-}}$-almost all $\gamma \in \hat{G}$.

The proof is complete.

As a consequence of Theorem 2 and Proposition 3, we have the following generalization of Theorem A.

Theorem 4. Let $\Gamma=\left(\Omega, G, \theta^{(\alpha)}, m_{\Omega}\right)$ be a special dynamical system, and let $E$ be a Borel subset of $\mathbb{R}$. If $x \in \mathscr{B}^{\mathrm{s}}\left(C^{*}(\Gamma)\right)_{\mathrm{s} a}$ is such that, for $m_{\hat{G}^{-}}$almost all $\gamma \in \hat{G}, \lambda_{\gamma}^{\prime \prime}(x)$ has pure point spectrum with finite multiplicity over $E$, then, for $\mathbb{P}$-almost all $\omega \in \Omega, \kappa_{\omega}^{\prime \prime}(x)$ has purely continuous spectrum over $E$.

\section{Some Covariant Representations}

Let $\Gamma=(\Omega, G, \theta, \mathbb{P})$ be a dynamical system. A covariant representation of $\Gamma$ is a triple $(\mathfrak{H}, \pi, U)$ in which $\mathfrak{H}$ is a Hilbert space, $\pi$ is a *-representation of $C(\Omega)$ in $\mathfrak{H}$, and $U$ is a strongly continuous unitary representation of $G$ in $\mathfrak{H}$ such that, for each $F \in C(\Omega)$ and each $g \in G$,

$$
\pi\left(\widetilde{\theta}_{g} F\right)=U(g) \pi(F) U(-g) .
$$

With any covariant representation $(\mathfrak{H}, \pi, U)$ of $\Gamma$ there is associated a nondegenerate *-representation $\varrho_{\pi, U}$ of $C^{*}(\Gamma)$ in $\mathfrak{Y}$ uniquely determined by

$$
\varrho_{\pi, U}(x)=\int_{G} \pi\left(x_{g}\right) U(g) d m_{G}(g) \quad(x \in \mathscr{K}(\Omega \times G)),
$$

the integral being taken in the strong-operator topology. It turns out that every non-degenerate ${ }^{*}$-representation of $C^{*}(\Gamma)$ arises as the *-representation associated with a certain covariant representation of $\Gamma$ (cf. [6, Proposition 7.6.4]). We 
illustrate this fact by giving any of the *-representations $\kappa_{\omega}(\omega \in \Omega)$ and $\lambda_{\gamma}(\gamma \in \hat{\Gamma})$ the form of a*-representation associated with a covariant representation of $\Gamma$.

Given a function $f$ on a group $G$ and an element $a$ of $G$, let $T_{a} f$ be the translate of $f$ by $a$, that is,

$$
T_{a} f(b)=f(a+b) \quad(b \in G) .
$$

For each $\omega \in \Omega$, let $\pi_{\omega}$ be the *-representation of $C(\Omega)$ in $L^{2}(G)$ defined by

$$
\left(\pi_{\omega}(F) \varphi\right)(g)=F\left(\theta_{g}(\omega)\right) \varphi(g) \quad\left(F \in C(\Omega), \varphi \in L^{2}(G), g \in G\right) .
$$

Let $T$ be the strongly continuous unitary representation of $G$ in $L^{2}(G)$ given by

$$
T(g) \varphi=T_{g} \varphi \quad\left(\varphi \in L^{2}(G), g \in G\right) .
$$

Then, for each $\omega \in \Omega,\left(L^{2}(G), \pi_{\omega}, T\right)$ is a covariant representation of $\Gamma$ and $\kappa_{\omega}=\varrho_{\pi_{\omega}, T}$.

For each $\gamma \in \hat{G}$, let $U_{\gamma}$ be the strongly continuous unitary representation of $G$ in $L^{2}(\Omega)$ defined by

$$
U_{\gamma}(g) H=(g, \gamma) \tilde{\theta}_{g} H \quad\left(H \in L^{2}(\Omega), g \in G\right) .
$$

Let $\mathscr{P}$ be the *-representation of $C(\Omega)$ in $L^{2}(\Omega)$ given by

$$
\mathscr{P}(F) H=M_{F} H \quad\left(F \in C(\Omega), H \in L^{2}(\Omega)\right) .
$$

Then, for each $\gamma \in \hat{G},\left(L^{2}(\Omega), \mathscr{P}, U_{\gamma}\right)$ is a covariant representation of $\Gamma$ and $\lambda_{\gamma}=\varrho_{\mathscr{P}, U_{\gamma}}$.

For the remainder of the present section, let $\Gamma=\left(\Omega, G, \theta^{(\alpha)}, m_{\Omega}\right)$ be a special dynamical system. Let $b G$ be the Bohr compactification of $G, \beta$ be the canonical monomorphism from $G$ into $b G$, and $\eta$ be the homomorphism from $b G$ onto $\Omega$ such that $\alpha=\eta \circ \beta$ (cf. [25, Definition 14.7.3]).

Let $J_{\eta}: L^{2}(\Omega) \rightarrow L^{2}(b G)$ be the operator given by

$$
J_{\eta} F=F \circ \eta \quad\left(F \in L^{2}(\Omega)\right) .
$$

Since $m_{\Omega}(A)=m_{b G}\left(\eta^{-1}(A)\right)$ for any Borel subset $A$ of $\Omega$, it follows that $J_{\eta}$ is an isometry.

Let $\wp$ be the *-representation of $C(\Omega)$ in $L^{2}(b G)$ given by

$$
\wp(F) H=M_{F \circ \eta} H \quad\left(F \in C(\Omega), H \in L^{2}(b G)\right) .
$$

Let $\mathscr{U}$ be a strongly continuous unitary representation of $G$ in $L^{2}(b G)$ defined by

$$
\mathscr{U}(g) F=T_{\alpha(g)} J_{\eta} F \quad\left(F \in L^{2}(b G), g \in G\right) .
$$

Then $\left(L^{2}(b G), \wp, \mathscr{U}\right)$ is a covariant representation of $\Gamma$. As we shall see shortly, the associated ${ }^{*}$-representation $\varrho_{\wp, \mathscr{U}}$ contains information simultaneously about all the ${ }^{*}$-representations $\lambda_{\gamma}(\gamma \in \hat{\Gamma})$.

For each $\gamma \in \widehat{G}$, let $\chi_{\gamma}$ be the element of $\widehat{b G}$ such that

$$
\left(\beta(g), \chi_{\gamma}\right)=(g, \gamma) \quad(g \in G) .
$$

Of course, the set $\left\{\chi_{\gamma}: \gamma \in \widehat{G}\right\}$ coincides with $\widehat{b G}$, and as such forms an orthonormal basis of $L^{2}(b G)$. Since $\alpha(G)$ is dense in $\Omega, \hat{\alpha}$ is injective. For each $\xi \in \hat{\alpha}(\hat{\Omega})$, let $\zeta_{\xi}=\hat{\alpha}^{-1}(\xi)$; then, clearly, $\zeta_{\xi} \circ \eta=\chi_{\xi}$. For each $\gamma \in \widehat{G}$, let $\mathfrak{H}_{\gamma}$ be the closed linear subspace of $L^{2}(b G)$ spanned by $\left\{\chi_{\gamma+\xi}: \xi \in \hat{\alpha}(\hat{\Omega})\right\}$. Note that if $\gamma-\gamma^{\prime} \in \hat{\alpha}(\hat{\Omega})$, then $\mathfrak{S}_{\gamma}=\mathfrak{H}_{\gamma^{\prime}}$, and if $\gamma-\gamma^{\prime} \in \hat{G} \backslash \hat{\alpha}(\widehat{\Omega})$, then $\mathfrak{H}_{\gamma}$ and $\mathfrak{S}_{\gamma^{\prime}}$ are mutually orthogonal. Let $\mathscr{S}$ be a selector of the quotient group $\hat{G} / \hat{\alpha}(\hat{\Omega})$, that is, a subset of $\widehat{G}$ whose intersection with 
any coset of $\hat{\alpha}(\hat{\Omega})$ is a singleton. Plainly

$$
L^{2}(b G)=\bigoplus_{\gamma \in \mathscr{S}} \mathfrak{H}_{\gamma} .
$$

Given $x \in \mathscr{K}(\Omega \times \mathbb{R}), \gamma \in \hat{G}$, and $\xi \in \hat{\Omega}$, we have

$$
\varrho_{\wp, \mathscr{U}}(x) \chi_{\gamma+\xi}=M_{\chi_{\gamma}} J_{\eta} \lambda_{\gamma}(x) \zeta_{\xi} .
$$

Hence, for each $\gamma \in \hat{G}, \mathfrak{H}_{\gamma}$ is an invariant subspace for $\varrho_{\wp, \mathscr{U}}$ and the restriction of $\varrho_{\wp, \mathscr{U}}$ to $\mathfrak{S}_{\gamma}$ is unitarily equivalent to $\lambda_{\gamma}$. Accordingly, up to a unitary equivalence,

$$
\varrho_{\wp, \mathscr{U}}=\bigoplus_{\gamma \in \mathscr{S}} \lambda_{\gamma} \text {. }
$$

Note that this representation does not depend on the choice of the selector $\mathscr{S}$ as, in view of (7), $\lambda_{\gamma}$ and $\lambda_{\gamma^{\prime}}$ are unitarily equivalent whenever $\gamma-\gamma^{\prime} \in \hat{\Omega}$.

\section{A Remark}

Let $\Gamma$ be a special dynamical system of the form $\left(\Omega, \mathbb{R}, \theta^{(\alpha)}, m_{\Omega}\right)$, and $(\mathfrak{H}, \pi, U)$ be a covariant representation of $\Gamma$. Denote by $T$ the set of all complex numbers with unit modulus. Let $Y: \Omega \times \mathbb{R} \rightarrow \mathbb{T}$ be a continuous cocycle on $\Omega$, that is, a continuous function satisfying the cocycle relation

$$
Y(\omega, s+t)=Y(\omega, s) Y(\omega+\alpha(s), t) \quad(\omega \in \Omega, s, t \in \mathbb{R}) .
$$

Given $t \in \mathbb{R}$, let $Y_{t}$ be the element of $C(\Omega)$ defined by

$$
Y_{t}(\omega)=Y(\omega, t) \quad(\omega \in \Omega)
$$

For each $t \in \mathbb{R}$, set

$$
G_{\pi, U, Y}(t)=\pi\left(Y_{t}\right) U(t)
$$

In view of (12), (14), the unitarity and the norm continuity of the function $\mathbb{R} \ni t$ $\rightarrow Y_{t} \in C(\Omega)$, the mapping $G_{\pi, U, Y}\left(t \rightarrow G_{\pi, U, Y}(t)\right)$ is a strongly continuous unitary oneparameter group in $\mathfrak{H}$. By Stone's theorem (cf. [28, Corollary 9.9.2]), the infinitesimal generator of $G_{\pi, U, Y}$ has the form $i A_{\pi, U, Y}$, where $A_{\pi, U, Y}$ is self-adjoint. Clearly, $A_{\pi, U, Y}^{2}$ is self-adjoint, positive, and, as an easy application of the operational calculus for normal operators reveals, for each $\mu<0$, the resolvent $R\left(\mu, A_{\pi, U, Y}^{2}\right)$ of $A_{\pi, U, Y}^{2}$ at $\mu$ satisfies

$$
R\left(\mu, A_{\pi, U, Y}^{2}\right)=-\frac{1}{2 \sqrt{-\mu}} \int_{\mathbb{R}} e^{-\sqrt{-\mu}|s|} G_{\pi, U, Y}(s) d s=\varrho_{\pi, U}\left(m_{\mu, Y}\right),
$$

where $m_{\mu, Y}$ is the element of $L^{1}(\Gamma)_{\mathrm{sa}}$ given by

$$
m_{\mu, Y}(\omega, s)=-\frac{1}{2 \sqrt{-\mu}} e^{-\sqrt{-\mu}|s|} Y(\omega, s) \quad(\omega \in \Omega, s \in \mathbb{R}) .
$$
by

With each $Q \in C_{\mathbb{R}}(\Omega)$ there is associated the continuous cocycle $Y^{(Q)}$ on $\Omega$ given

$$
Y^{(Q)}(\omega, t)=\exp \left(i \int_{0}^{t} Q(\omega+\alpha(s)) d s\right) \quad(\omega \in \Omega, t \in \mathbb{R})
$$


If we denote by $D_{U}$ the infinitesimal generator of the unitary one-parameter group $U$, then, as one directly verifies,

$$
A_{\pi, U, Y^{(Q)}}=i^{-1} D_{U}+\pi(Q) .
$$

A fundamental fact is that there exist functions $Q$ in $C_{\mathbb{R}}(\Omega)$ such that, for each $\gamma \in \widehat{\mathbb{R}}, A_{\mathscr{P}, U_{\gamma}, Y(Q)}$ has purely continuous spectrum. More precisely, there exist functions $Q$ in $C_{\mathbb{R}}(\Omega)$ such that, for each $\gamma \in \hat{\mathbb{R}}, A_{\mathscr{P}, U_{\gamma}, Y^{(Q)}}$ has purely Lebesgue spectrum; and there exist functions $Q$ in $C_{\mathbb{R}}(\Omega)$ such that, for each $\gamma \in \widehat{\mathbb{R}}, A_{\mathscr{P}, U_{\gamma}, Q}$ has purely singularly continuous spectrum. The truth of the fact is seen as follows. Let $(\Theta, \mathfrak{M}, \mu)$ be a probability space carrying a sequence $\left(X_{n}\right)_{n \in \mathbb{N}}$ of $\Omega$-valued independent random variables, each uniformly distributed on $\Omega$. Let $f$ be a unitary continuous function on $\mathbb{T}$ with at least two non-zero Fourier coefficients. Then, by a result of [11], there exists a sequence $\left(\zeta_{n}\right)_{n \in \mathbb{N}}$ in $\hat{\Omega}$ with $\left(\hat{\alpha}\left(\zeta_{n}\right)\right)_{n \in \mathbb{N}}$ tending to 0 as fast as we please such that, for each $(\theta, \omega, t) \in \Theta \times \Omega \times \mathbb{R}$, the product

$$
\prod_{n=1}^{\infty} f\left(\left(\omega+X_{n}(\theta), \zeta_{n}\right)\right) \overline{f\left(\left(\omega+X_{n}(\theta)+\alpha(t), \zeta_{n}\right)\right)}
$$

converges (with uniform convergence in $\theta$ and $\omega$, and with local uniform convergence in $t$ ) and, for any fixed $\theta \in \Theta$, defines a continuous cocycle $Y_{\theta, f}$ on $\Omega$ such that, for $\mu$-almost all $\theta \in \Theta$, all the operators $A_{\mathscr{P}, U_{\gamma}, Y_{\theta, f}}(\gamma \in \widehat{\mathbb{R}})$ have purely Lebesgue spectrum (respectively purely singularly continuous spectrum). Let $g$ be a real non-constant continuous function on $\mathbb{T}$ such that $(2 \pi)^{-1} \int_{0}^{2 \pi} g\left(e^{i u}\right) d u$ is an integer, and, for each $s \in[0,2 \pi)$, set

$$
f\left(e^{i s}\right)=\exp \left(-i \int_{0}^{s} g\left(e^{i u}\right) d u\right) .
$$

Then $f$ is a unitary continuous function on $\mathbb{T}$ with at least two non-zero Fourier coefficients. Now, as indicated above, one can choose a sequence $\left(\zeta_{n}\right)_{n \in \mathbb{N}}$ in $\hat{\Omega}$ so that, if, for each $n \in \mathbb{N}, \alpha_{n}$ is such that

$$
e^{i \alpha_{n} t}=\left(\alpha(t), \zeta_{n}\right) \quad(t \in \mathbb{R}),
$$

then $\sum_{n=1}^{\infty}\left|\alpha_{n}\right|<+\infty$ and, if, for each $\theta \in \Theta$, the function $Q_{\theta}$ in $C(\Omega)$ is given by

$$
Q_{\theta}(\omega)=\sum_{n=1}^{\infty} \alpha_{n} g\left(\left(\omega+X_{n}(\theta), \zeta_{n}\right)\right) \quad(\omega \in \Omega),
$$

then $Y_{\theta, f}=Y^{\left(Q_{\theta}\right)}$ and, for $\mu$-almost all $\theta \in \Theta$, all the operators $A_{\mathscr{P}, U_{\gamma}, Y^{\left(Q^{\theta}\right)}}(\gamma \in \widehat{\mathbb{R}})$ have purely Lebesgue spectrum (respectively purely singularly continuous spectrum).

Note that, for each $Q \in C_{\mathbb{R}}(\Omega)$ and each $\omega \in \Omega, A_{\pi_{\omega}, T, Y(Q)}$ coincides with the operator $i^{-1}(d / d x)+q_{\omega}(x)$, defined on the Sobolev space $H^{1}(\mathbb{R})$, where $q_{\omega}=\left(T_{\omega} Q\right) \circ \alpha$. For each $x \in \mathbb{R}$, set

$$
u_{\omega, Q}(x)=\exp \left(-i \int_{0}^{x} q_{\omega}(s) d s\right) .
$$

It is readily verified that

$$
M_{u_{\omega, Q}} A_{\pi_{\omega}, T, Q} M_{u_{\omega, Q}}^{-1}=\frac{1}{i} \frac{d}{d x},
$$


so that $A_{\pi_{\omega}, T, Y(Q)}$ and $i^{-1}(d / d x)$ are unitarily equivalent. Accordingly, $A_{\left.\pi_{\omega}, T, Y^{(}()\right)}$ has purely Lebesgue spectrum.

Let $Q \in C_{\mathbb{R}}(\Omega)$ be such that, for each $\gamma \in \mathbb{R}, A_{\mathscr{P}, U_{\gamma}, Y^{(Q)}}$ has purely continuous spectrum. Fix arbitrarily $\mu<0$. Then, for each $\omega \in \Omega, A_{\pi_{\omega}, T, Y(Q)}^{2}$ has purely Lebesgue spectrum and hence, by (15), so does $\kappa_{\omega}\left(m_{\mu, Y(Q)}\right)$. Moreover, for each $\gamma \in \widehat{\mathbb{R}}, A_{\mathscr{P}, U_{\gamma}, Q}$ has purely continuous spectrum, and so, by $(15), \lambda_{\gamma}\left(m_{\left.\mu, Y^{(Q)}\right)}\right)$ has purely continuous spectrum. We thus see there exist elements of $C^{*}(\Gamma)_{\mathrm{sa}}$ whose images by the $\kappa_{\omega}(\omega \in \Omega)$ have purely continuous spectrum without the images by the $\lambda_{\gamma}(\gamma \in \mathbb{R})$ having pure point spectrum.

\section{Some Applications}

5.1. Consider $\mathbb{T}$ as a compact group with multiplication as group operation, and let $\Gamma=\left(\mathbb{T}, \mathbb{Z}, \theta^{(\alpha)}, m_{\mathbb{T}}\right)$ be a special dynamical system in which the homomorphism $\alpha: \mathbb{Z} \rightarrow \mathbb{T}$ is given by

$$
\alpha(n)=e^{2 \pi i \xi n} \quad(n \in \mathbb{Z})
$$

with $\xi \in \mathbb{R} \backslash \mathbb{Q}$. Let $u$ and $v$ be the elements of $\mathscr{K}(\mathbb{T} \times \mathbb{Z})$ defined by

$$
u(\omega, n)=1_{\{1\}}(n) \text { and } v(\omega, n)=\omega 1_{\{0\}}(n) \quad(\omega \in \mathbb{T}, n \in \mathbb{Z}) .
$$

Considered as elements of $C^{*}(\Gamma), u$ and $v$ are unitaries satisfying the twisted commutation relation

$$
u \circ v=e^{2 \pi i \xi} v \circ u
$$

A direct computation shows that $C^{*}(\Gamma)$ coincides with the $C^{*}$-algebra generated by $u$ and $v$. It is well known that there exists exactly one, up to *-isomorphism, $C^{*}$ algebra generated by two unitaries satisfying the above twisted commutation relation (cf. $\left[7 ; 26\right.$, p. 117]). That $C^{*}$-algebra is called the irrational rotation algebra and is usually denoted by $\mathscr{A}_{\xi}$. Accordingly, $C^{*}(\Gamma)$ is a realisation of $\mathscr{A}_{\xi}$.

Given an operator $T$ in a Banach space or an element $T$ of a Banach algebra, denote by $\sigma(T)$ the spectrum of $T$.

Since $C^{*}(\Gamma)$ is simple (cf. [26, Theorem 4.3.3]), all the *-representations of $C^{*}(\Gamma)$ are faithful. Hence, in particular, $\sigma\left(\kappa_{\omega}(x)\right)=\sigma(x)$ for each $x \in C^{*}(\Gamma)$ and each $\omega \in \mathbb{T}$.

Given a *-algebra $A$, let $\operatorname{Aut}(A)$ be the group of all *-automorphisms of $A$. For a subset $E$ of $A$ and $\mathfrak{a} \in \operatorname{Aut}(A)$, let $E^{\mathfrak{a}}$ be the set of all fixed points of $\mathfrak{a}$ in $E$. For each $\mathfrak{a} \in \operatorname{Aut}(A)$ and each $x \in A$, let $\mathfrak{a}^{0}(x)=x$ and, by induction, let $\mathfrak{a}^{n}(x)=\mathfrak{a}\left(\mathfrak{a}^{n-1}(x)\right)$ for each $n \in \mathbb{N}$. If $\mathfrak{a} \in \operatorname{Aut}(A)$ is such that $\mathfrak{a}^{n}=\mathrm{id}_{A}$ for some $n \in \mathbb{N}$, then setting

$$
\pi_{a}(x)=\frac{1}{n} \sum_{k=0}^{n-1} \mathfrak{a}^{k}(x) \quad(x \in A)
$$

defines a projection $\pi_{\mathfrak{a}}$ from $A$ onto $A^{\mathfrak{a}}$.

Let $K$ be the *-subalgebra of $C^{*}(\Gamma)$ generated by $u$ and $v$. Clearly, if $\mathfrak{a} \in \operatorname{Aut}\left(C^{*}(\Gamma)\right)$ is such that $\mathfrak{a}^{n}=\mathrm{id}_{C^{*}(\Gamma)}$ for some $n \in \mathbb{N}$, then $\pi_{\mathrm{a}}$ maps $K_{\text {sa }}$ onto $K_{\mathrm{sa}}^{a}$.

For any $s \in \operatorname{SL}(2, \mathbb{Z})$ and any $m, n \in \mathbb{Z}$, denote by $\left(m_{s}, n_{s}\right)$ the image of $(m, n)$ under the standard action of $s$ on $\mathbb{Z} \times \mathbb{Z}$. As shown by Brenken [7], the exists a representation $s \rightarrow \mathfrak{a}_{s}$ of $\operatorname{SL}(2, \mathbb{Z})$ in $\operatorname{Aut}\left(C^{*}(\Gamma)\right)$ such that

$$
\mathfrak{a}_{s}\left(v^{m} u^{n}\right)=e^{\pi i \xi\left(m_{s} n_{s}-m n\right)} v^{m_{s}} u^{n_{s}} \quad(s \in \operatorname{SL}(2, \mathbb{Z}), m, n \in \mathbb{Z}) .
$$


Let

$$
s_{0}=\left(\begin{array}{rr}
0 & -1 \\
1 & 0
\end{array}\right) .
$$

In the sequel, the automorphism $\mathfrak{a}_{s_{0}}$ will play a special rôle and will be denoted briefly as $\mathfrak{b}$. It is easy to see that $\mathfrak{b}$ is uniquely determined by the identities

$$
\mathfrak{b}(u)=v^{*} \quad \text { and } \quad \mathfrak{b}(v)=u .
$$

Obviously, as $s_{0}^{4}=e$, where $e$ is the neutral element of $\operatorname{SL}(2, \mathbb{Z})$, we have $\mathfrak{b}^{4}=\mathrm{id}_{C^{*}(\Gamma)}$.

One of the elements of $K_{\mathrm{sa}}^{\mathrm{b}}$ is

$$
h=2 \pi_{\mathrm{b}}\left(u+u^{*}\right)=u+u^{*}+v+v^{*} .
$$

The corresponding operators $\kappa_{\omega}(h)(\omega \in \mathbb{T})$ arise as hamiltonians in the Harper model of a two-dimensional crystal with square symmetry in a magnetic field. If $\omega=e^{2 \pi i \theta}$ with $\theta \in[0,1)$, then, as one easily verifies,

$\left(\kappa_{\omega}(h) \varphi\right)(n)=\varphi(n+1)+\varphi(n-1)+2 \cos 2 \pi(\theta+\xi n) \varphi(n), \quad\left(\varphi \in l^{2}(\mathbb{Z})=L^{2}(\mathbb{Z}), n \in \mathbb{Z}\right)$.

The spectral properties of the $\kappa_{\omega}(h)(\omega \in \mathbb{T})$ and of related operators have long been investigated by physicists and mathematicians (cf. [1, 13-17, 27] and the bibliographies therein). A still unproved conjecture asserts that, for each $\omega \in \mathbb{T}$, $\kappa_{\omega}(h)$ has purely singular continuous spectrum and that $\sigma(h)$, which, as indicated above, coincides with $\sigma\left(\kappa_{\omega}(h)\right)$ for each $\omega \in \mathbb{T}$, is of zero Lebesgue measure. Using an argument due to Aubry and André ([1]; cf. also [2, 4]), we shall establish a result (Theorem 6) concerning the entire space $K_{\text {sa }}^{\mathrm{b}}$, which, when applied to $h$, partially substantiates the conjecture.

Theorem 5. For every $x \in K_{\mathrm{sa}}^{\mathrm{b}}$ and every Borel subset $E$ of $\mathbb{R}$, either $\left(\kappa_{\omega}(x)\right)_{E}=0$ for $m_{\mathbb{T}^{-}}$almost all $\omega \in \mathbb{T}$, or, for $m_{\mathbb{T}^{-}}$almost all $\omega \in \mathbb{T}, \kappa_{\omega}(x)$ has no pure point spectrum over $E$.

Proof. Let $\mathscr{F}$ be the Fourier transformation from $L^{2}(\mathbb{T})$ onto $l^{2}(\mathbb{Z})$ given by

$$
(\mathscr{F} F)(n)=\int_{\mathbb{T}} F(\omega) \bar{\omega}^{n} d m_{\mathbb{T}}(\omega) \quad\left(F \in L^{2}(\mathbb{T})\right) .
$$

As is well known, $\mathscr{F}$ sets up a unitary equivalence between $L^{2}(\mathbb{T})$ and $l^{2}(\mathbb{Z})$. Identifying $\hat{\mathbb{Z}}$ with $\mathbb{T}$ in a standard way, one directly verifies that for each $\omega \in \mathbb{T}$, each $\varphi \in l^{2}(\mathbb{Z})$, and each $n \in \mathbb{Z}$,

$$
\begin{aligned}
& \left(\kappa_{\omega}(u) \varphi\right)(n)=\left(\mathscr{F} \lambda_{\omega}\left(v^{*}\right) \mathscr{F}^{-1} \varphi\right)(n)=\varphi(n+1), \\
& \left(\kappa_{\omega}(v) \varphi\right)(n)=\left(\mathscr{F} \lambda_{\omega}(u) \mathscr{F}^{-1} \varphi\right)(n)=\omega e^{2 \pi i \xi n} \varphi(n) .
\end{aligned}
$$

Consequently, for each $y \in C^{*}(\Gamma)$ and each $\omega \in \mathbb{T}$,

$$
\kappa_{\omega}(y)=\mathscr{F} \lambda_{\omega}(\mathfrak{b}(y)) \mathscr{F}^{-1} .
$$

Now, if $x \in K_{\mathrm{sa}}^{\mathrm{b}}$ and $\omega \in \mathbb{T}$, then

$$
\kappa_{\omega}(x)=\mathscr{F} \lambda_{\omega}(x) \mathscr{F}^{-1} .
$$

Hence, for each $\omega \in \mathbb{T}$ and each bounded continuous function $f$ on $\mathbb{R}$,

$$
\kappa_{\omega}(f(x))=\mathscr{F} \lambda_{\omega}(f(x)) \mathscr{F}^{-1},
$$


and further, by the sequential normality of $\kappa_{\omega}^{\prime \prime}$ and $\lambda_{\omega}^{\prime \prime}$, for each bounded Borel function $f$ on $\mathbb{R}$,

$$
\kappa_{\omega}^{\prime \prime}(f(x))=\mathscr{F} \lambda_{\omega}^{\prime \prime}(f(x)) \mathscr{F}^{-1} .
$$

In particular, for each $\omega \in \mathbb{T}$ and each Borel subset $E$ of $\mathbb{R}$,

$$
\kappa_{\omega}^{\prime \prime}\left(x_{E}\right)=\mathscr{F} \lambda_{\omega}^{\prime \prime}\left(x_{E}\right) \mathscr{F}^{-1} .
$$

It is also easy to see that, for each $\omega \in \mathbb{T}$,

$$
\kappa_{\theta^{(\alpha)}(\omega, n)}(x)=T(n) \kappa_{\omega}(x) T(-n),
$$

whence, by a similar argument, for each $\omega \in \mathbb{T}$ and each Borel subset $E$ of $\mathbb{R}$,

$$
\kappa_{\theta^{(\alpha)}(\omega, n)}^{\prime \prime}\left(x_{E}\right)=T(n) \kappa_{\omega}^{\prime \prime}\left(x_{E}\right) T(-n)
$$

where, of course, $x_{E}$ denotes the element $1_{E}(x) x$ of $\mathscr{B}^{s}\left(C^{*}(\Gamma)\right)_{\mathrm{sa}}$. Since the function $\omega \rightarrow \kappa_{\omega}(x)$ is strongly continuous, it follows, by a standard argument, that for every bounded Borel function $f$ on $\mathbb{R}$ the function $\omega \rightarrow \kappa_{\omega}^{\prime \prime}(f(x))$ is weakly Borel measurable. In particular, for each Borel subset $E$ of $\mathbb{R}$, the function $\omega \rightarrow \kappa_{\omega}^{\prime \prime}\left(x_{E}\right)$ is weakly Borel measurable. Now, since the dynamical system $\Gamma$ is ergodic, it follows from a theorem of Kunz-Soullaird ([22]; cf. also [20]) that, for each Borel subset $E$ of $\mathbb{R}$, the set of those $\omega \in \mathbb{T}$ for which $\kappa_{\omega}^{\prime \prime}\left(x_{E}\right)$ has pure point spectrum is either $m_{\mathbb{T}^{-}}$ null or of full measure in $\mathbb{T}$.

Suppose that, for some Borel subset $E$ of $\mathbb{R}$, the set of those $\omega \in \mathbb{T}$ for which $\kappa_{\omega}(x)$ has no pure point spectrum over $E$ is not of full measure in TI. Since, for each $\omega \in \mathbb{T}, \kappa_{\omega}(x)$ has pure point spectrum over $E$ if and only if $\left(\kappa_{\omega}(x)\right)_{E}=\kappa_{\omega}^{\prime \prime}\left(x_{E}\right)$ has pure point spectrum, it follows from the preceding paragraph that, for $m_{\mathbb{T}}$-almost all $\omega \in \mathbb{T}, \kappa_{\omega}^{\prime \prime}\left(x_{E}\right)$ has pure point spectrum. Now, by $(18), \lambda_{\omega}^{\prime \prime}\left(x_{E}\right)$ has also pure point spectrum for $m_{\mathbb{T}}$-almost all $\omega \in \mathbb{T}$, and hence $\lambda_{\omega}(x)$ has pure point spectrum over $E$ for $m_{\mathbb{T}}$-almost all $\omega \in \mathbb{T}$. In view of (16), for each $\omega \in \mathbb{T}, \kappa_{\omega}(x)$ is a difference operator of finite order, and so every eigenvalue of $\kappa_{\omega}(x)$ has finite multiplicity. Accordingly, by (17), for each $\omega \in \mathbb{T}$, every eigenvalue of $\lambda_{\omega}(x)$ has finite multiplicity. Applying now Theorem 4 , we find that, for $m_{\mathbb{T}}$-almost all $\omega \in \mathbb{T}, \kappa_{\omega}(x)$ has purely continuous spectrum over $E$. Finally, the fact that, for $m_{\mathbb{T}}$-almost all $\omega \in \mathbb{T}, \kappa_{\omega}(x)$ has simultaneously pure point and purely continuous spectrum over $E$ implies that $\kappa_{\omega}^{\prime \prime}\left(x_{E}\right)=0$ for $m_{\mathbb{T}}$-almost all $\omega \in \mathbb{T}$.

The proof is complete.

Theorem 6. For every $x \in K_{\mathrm{sa}}^{\mathrm{b}}$ and $m_{\mathbb{T}}$-almost all $\omega \in \mathbb{T}, \kappa_{\omega}(x)$ does not have pure point spectrum over any non-empty open subset of $\sigma(x)$.

Proof. Let $x \in K_{\mathrm{sa}}^{\mathrm{b}}$. Since the topology of $\sigma(x)$ has a countable basis, it suffices to prove that, for each non-empty open subset of $\sigma(x)$ and for $m_{\mathbb{T}}$-almost all $\omega \in \mathbb{T}$, $\kappa_{\omega}(x)$ has no pure point spectrum over that subset.

Let $U$ be a non-empty open subset of $\sigma(x)$ and $f: \mathbb{R} \rightarrow[0,1]$ be a non-zero continuous function with support in $U$. Then $0<f(x) x^{2} \leqq x_{U}^{2}$. Hence, by the faithfulness of the $\kappa_{\omega}(\omega \in \mathbb{T})$, for each $\omega \in \mathbb{T}, 0<\kappa_{\omega}\left(f(x) x^{2}\right) \leqq\left(\kappa_{\omega}^{\prime \prime}\left(x_{U}\right)\right)^{2}$ and so $\kappa_{\omega}^{\prime \prime}\left(x_{U}\right) \neq 0$. Now the theorem follows upon applying Theorem 5 .

5.2. Let $\Gamma=\left(\Omega, \mathbb{R}, \theta^{(\alpha)}, m_{\mathbb{R}}\right)$ be a special dynamical system, $(\mathfrak{H}, \pi, U)$ be a covariant representation of $\Gamma$, and $D_{U}$ be the infinitesimal generator of $U$. Then $-D_{U}^{2}$ is selfadjoint, positive, and, for each $\mu<0$,

$$
R\left(\mu,-D_{U}^{2}\right)=-\frac{1}{2 \sqrt{-\mu}} \int_{\mathbb{R}} e^{-V-\mu|s|} U(s) d s .
$$


Given $Q \in C_{\mathbb{R}}(\Omega)$, let $H_{\pi, U, Q}$ be the self-adjoint operator defined by

$$
H_{\pi, U, Q}=-D_{U}^{2}+\pi(Q)
$$

with domain coinciding with that of $D_{U}^{2}$. Clearly, $H_{\pi, U, Q}$ is self-adjoint and bounded below by $-\|Q\|_{\infty}$.

For each $\mu<0$ and each $F \in C(\Omega)$, let $x_{\mu, F}$ be the element of $L^{1}(\Gamma)$ given by

$$
x_{\mu, F}(\omega, s)=-\frac{1}{2 \sqrt{-\mu}} e^{-V-\mu|s|} T_{\alpha(s)} F \quad(\omega \in \Omega, s \in \mathbb{R}) .
$$

Clearly, $\left\|x_{\mu, F}\right\|_{1}=\|F\|_{\infty} /|\mu|$. Moreover, in view of (12) and (19),

$$
R\left(\mu,-D_{U}^{2}\right) \pi(F)=\varrho_{\pi, U}\left(x_{\mu, F}\right) .
$$

Since, for $\mu<-\|Q\|_{\infty}$,

$$
\left\|x_{\mu, 1}\right\|_{1}+\left\|x_{\mu, 1}\right\|_{1} \sum_{n=1}^{\infty}\left\|x_{\mu, Q}\right\|_{1}^{n}=\frac{1}{|\mu|}+\frac{1}{|\mu|} \sum_{n=1}^{\infty}\left(\frac{\|Q\|_{\infty}}{|\mu|}\right)^{n}=\frac{1}{|\mu|-\|Q\|_{\infty}},
$$

it follows that the series

$$
x_{\mu, 1}+x_{\mu, 1} \circ \sum_{n=1}^{\infty}\left(x_{\mu, Q}\right)^{\circ n}
$$

converges in $L^{1}(\Gamma)$. Let $r_{\mu, F}$ be its sum. Since, for $\mu<-\|Q\|_{\infty}$,

$$
R\left(\mu, H_{\pi, U, Q}\right)=R\left(\mu,-D_{U}^{2}\right)\left(I+\sum_{n=1}^{\infty}\left(R\left(\mu,-D_{U}^{2}\right) \pi(Q)\right)^{n}\right),
$$

it follows from (20) that

$$
R\left(\mu, H_{\pi, U, Q}\right)=\varrho_{\pi, U}\left(r_{\mu, Q}\right) .
$$

A moment's reflection shows that $r_{\mu, Q}$ is self-adjoint.

The argument used in the proof of (21) goes back to Bellissard and Testard [5] (see also [3, Theorem 3.1]).

Note that, for each $\omega \in \Omega, H_{\pi_{\omega}, T, Q}$ is the Schrödinger operator $\left(-d^{2} / d x^{2}\right)$ $+q_{\omega}(x)$ with the almost periodic potential $q_{\omega}=\left(T_{\omega} Q\right) \circ \alpha$, defined on the Sobolev space $H^{2}(\mathbb{R})$. Each $H_{\mathscr{P}, U_{\gamma}, Q}(\gamma \in \hat{\mathbb{R}})$ is a so-called Bloch operator. The operator $H_{\wp, \varkappa, Q}$ was first introduced and studied by Burnat ([8]; cf. also $\left.[9,10,18,21]\right)$ and we shall accordingly call $H_{f}, \mathfrak{u}, Q$ the Burnat operator.

The main result of this subsection is the following.

Theorem 7. Let $\Gamma=\left(\Omega, \mathbb{R}, \theta^{(\alpha)}, m_{\Omega}\right)$ be a special dynamical system, let $Q$ be an element of $C_{\mathbb{R}}(\Omega)$, and let $E$ be a Borel subset of $\mathbb{R}$. If, for $m_{\hat{\mathbb{R}}}$-almost all $\gamma \in \hat{\mathbb{R}}$, the Bloch operator $H_{\mathscr{P}, U_{\gamma}, Q}$ has pure point spectrum over $E$, then, for $m_{\Omega}$-almost all $\omega \in \Omega$, the Schrödinger operator $H_{\pi_{\omega}, T, Q}$ has purely continuous spectrum over $E$.

Proof. Fix arbitrarily $\mu<-\|Q\|_{\infty}$. Let

$$
F=\left\{f \in \mathbb{R}: f=(\mu-e)^{-1}, e \in E\right\} .
$$

In view of (21), for $m_{\mathbb{R}^{-}}$-almost all $\gamma \in \mathbb{R}, \lambda_{\gamma}^{\prime \prime}\left(r_{\mu, Q}\right)$ has pure point spectrum over $F$. By the result of [12], every eigenvalue of the Burnat operator $H_{\wp, \mathscr{Q}, Q}$ is at most double. Hence, in view of (20), every eigenvalue of $\varrho_{\varnothing, \vartheta}^{\prime \prime}\left(r_{\mu, Q}\right)$ is at most double. Now, by (13), every eigenvalue of $\lambda_{\gamma}^{\prime \prime}\left(r_{\mu, Q}\right)$ is at most double whatever $\gamma \in \hat{\mathbb{R}}$. By 
virtue of Theorem 4 , for $m_{\Omega}$-almost all $\omega \in \Omega, \kappa_{\omega}^{\prime \prime}\left(r_{\mu, Q}\right)$ has purely continuous spectrum over $F$, and hence, for $m_{\Omega}$-almost all $\omega \in \Omega, H_{\pi_{\omega}, T, Q}$ has purely continous spectrum over $E$.

The proof is complete.

\section{References}

1. Avron, J., v. Mouche, P.H.M., Simon, B.: On the measure of the spectrum for the almost Mathieu operator. Commun. Math. Phys. 132, 103-118 (1990)

2. Bellissard, J.: Schrödinger operators with almost periodic potential: an overview. In: Schrader, R., Seiler, R., Uhlenbrok, D.A. (eds.) Mathematical problems in theoretical physics (Berlin, 1981), pp. 356-363. Lecture Notes in Phys. vol. 153. Berlin, New York: Springer 1982

3. Bellissard, J., Lima, D., Testard, D.: Almost periodic Schrödinger operators. In: Streit, L. (ed.) Mathematics and Physics, Lectures on Recent Results, vol.1, pp. 1-64. Singapore, Philadelphia: World Scientific 1985

4. Bellissard, J., Testard, D.: Quasi-periodic Hamiltonians. A mathematical approach. In: Kadison, R.V. (ed.) Operator algebras and applications, Part 2 (Kingston, Ontario, 1980), pp. 297-299. Proc. Sympos. Pure Math. 38, Providence, R.I.: Am. Math. Soc. 1982

5. Bellissard, J., Testard, D.: Almost periodic hamiltonians: An algebraic approach, Preprint CPT-81/P. 1311, Université de Provence, Marseille

6. Bratelli, G.: $C^{*}$-algebras and their automorphism groups. London, New York, San Francisco: Academic Press 1979

7. Brenken, B.A.: Representations and automorphisms of the irrational rotation algebra. Pacific J. Math. 111, 257-282 (1984)

8. Burnat, M.: Die Spektraldarstellung einiger Differentialoperatoren mit periodischen Koeffizienten im Raume der fastperiodischen Funktionen. Studia Math. 25, 33-64 (1964)

9. Burnat, M.: The spectral properties of the Schrödinger operator in nonseparable Hilbert spaces. Banach Center Publ. 8, 49-56 (1982)

10. Chojnacki, W.: Spectral analysis of Schrödinger operators in non-separable Hilbert spaces. Functional integration with emphasis on the Feynman integral (Sherbrooke, PQ, 1986). Rend. Circ. Mat. Palermo (2) [Suppl.] 17, 135-151 (1987)

11. Chojnacki, W.: Some non-trivial cocycles. J. Funct. Anal. 77, 9-31 (1988)

12. Chojnacki, W.: Eigenvalues of almost periodic Schrödinger operator in $L^{2}(b \mathbb{R})$ are at most double. Lett. Math. Phys. 22, 7-10 (1991)

13. Delyon, F.: Absence of localisation in the almost Mathieu equation. J. Phys. A 20, L21-L23 (1987)

14. Helffer, B., Sjöstrand, J.: Analyse semi-classique pour l'équation de Harper. Mém. Soc. Math. France (N.S.) 34, 1-113 (1988)

15. Helffer, B., Sjöstrand, J.: Semi-classical analysis for Harper's equation. III. Mém. Soc. Math. France (N.S.) 39, 1-124 (1989)

16. Helffer, B., Sjöstrand, J.: Analyse semi-classique pour l'équation de Harper. II. Mém. Soc. Math. France (N.S.) 40, 1-139 (1990)

17. Helffer, B., Kerdelhué, P., Sjöstrand, J.: Le papillon de Hofstadter revisité. Mém. Soc. Math. France (N.S.) 43, 1-87 (1990)

18. Herczyński, J.: Schrödinger operators with almost periodic potentials in nonseparable Hilbert spaces. Banach Center Publ. 19, 121-142 (1987)

19. Kaminker, J., Xia, J.: The spectrum of operators elliptic along the orbits of $\mathbb{R}^{n}$ actions. Commun. Math. Phys. 110, 427-438 (1987)

20. Kirsch, W., Martinelli, F.: On the ergodic properties of the spectrum of general random operators. J. Reine Angew. Math. 334, 141-156 (1982)

21. Krupa, A., Zawisza, B.: Ultrapowers of unbounded selfadjoint operators. Studia Math. 85, 107-123 (1987)

22. Kunz, H., Souillard, B.: Sur le spectre des opérateurs aux differences finies aléatoires. Commun. Math. Phys. 78, 201-246 (1980)

23. Morris, S.: Pontryagin duality and the structure of locally compact abelian groups. Cambridge: Cambridge University Press 1977 
24. Rudin, W.: Fourier analysis on groups. New York: Interscience 1962

25. Semadeni, Z.: Banach spaces of continuous spaces, vol. 1. Warszawa: PWN 1971

26. Tomiyama, J.: Invitation to $C^{*}$-algebras and topological dynamics. Singapore, New Jersey, Hong Kong: World Scientific 1987

27. Wilkinson, M.: Critical properties of electron eigenstates in incommensurate systems. Proc. R. Soc. London Ser. A 391, 305-350 (1984)

28. Yosida, K.: Functional analysis. Berlin, Heidelberg, New York: Springer 1980

29. Żelazko, W.: Banach algebras. Amsterdam, London, New York: Elsevier, Warszawa: PWN 1973

Communicated by H. Araki 